\title{
Berberina, curcumina y quercetina como potenciales agentes con capacidad antiparasitaria
}

\author{
Joel H. Elizondo-Luévano ${ }^{1 *}$, Magda E. Hernández-García ${ }^{1}$, Oscar A. Pérez-Narváez ${ }^{1}$, \\ Rocío Castro-Ríos ${ }^{2} \&$ Abelardo Chávez-Montes ${ }^{1 *}$ \\ 1. Departamento de Química, Facultad de Ciencias Biológicas, Universidad Autónoma de Nuevo León, Ciudad \\ Universitaria, C.P. 66455, San Nicolás de los Garza, Nuevo León, México; joel.elizondolv@uanl.edu.mx, \\ magda.hernandezgr@uanl.edu.mx, oscar.pereznr@uanl.edu.mx, abelardo.chavezmn@uanl.edu.mx \\ 2. Departamento de Química, Facultad de Medicina, Universidad Autónoma de Nuevo León, C.P. 64460, Monterrey, \\ Nuevo León, México; rocio.castrors@uanl.edu.mx \\ * Correspondencia
}

Recibido 30-V-2020. Corregido 15-VII-2020. Aceptado 26-VIII-2020.

\begin{abstract}
Berberine, curcumin and quercetin as potential antiparasitic agents. Introduction: The indiscriminate use of antiparasitic agents has resulted in the establishment of resistance to them. Therefore, the development of new treatment alternatives is necessary. Natural products have various qualities as possible adjuvants in therapies against different etiological agents, among which its antiparasitic effects stand out. Objective: To evaluate the antiparasitic, antioxidant, cytotoxic, and cytoprotective activity of Berberine (Ber), Curcumin (Cur), and Quercetin (Qr). Methods: Analytical grade Ber, Cur, and Qr solutions were prepared, and aliquots were made at different concentrations for their evaluation against Entamoeba histolytica, Trichomonas vaginalis, and Strongyloides venezuelensis. To do this, the mean inhibitory concentration $\left(\mathrm{IC}_{50}\right)$ was determined, and the antioxidant capacity $\left(\mathrm{EC}_{50}\right)$ was also determined by the DPPH assay, both using the Probit statistical test. The cytotoxic and cytoprotective activity was determined by the hemolysis technique, Anova and Tukey's test were applied to determine the difference in the means in the treatments evaluated. Results: Ber, Cur, and Qr, showed activity against E. histolytica, $T$. vaginalis, and $S$. venezuelensis in-vitro. Ber presented $\mathrm{IC}_{50}$ of 1.7 , 1.2 , and $1.9 \mu \mathrm{M}$ respectively, being more effective compared to Cur with $\mathrm{IC}_{50}$ of 55.3, 40.6, and $13.7 \mu \mathrm{M}$, or $\mathrm{Qr}$ with $\mathrm{IC}_{50}$ of $147.2,93.2$, and $110.9 \mu \mathrm{M}$, however, the best antioxidant activity $\left(\mathrm{EC}_{50}=1.1 \mu \mathrm{g} / \mathrm{ml}\right)$, cytoprotective and less hemolytic, was presented by $\mathrm{Qr}(\mathrm{P}<0.001)$ compared to the evaluated control. Conclusions: The metabolites of natural origin berberine, curcumin, and quercetin, have activity against trophozoites of $E$. histolytica, T. vaginalis and larvae of $S$. venezuelensis in low doses comparable to the reference drugs in the case of Ber. Furthermore, these non-synthetic products of natural origin could be the subject of future research to help treat parasitosis, since in low doses, they showed antioxidant activity without showing considerable cytotoxicity in human erythrocytes.
\end{abstract}

Key words: antiparasitic activity; antiparasitics; berberine; curcumin; quercetin.

Elizondo-Luévano, J.H., Hernández-García, M.E., Pérez-Narváez, O.A., Castro-Ríos, R., \& Chávez-Montes, A. (2020). Berberina, curcumina y quercetina como potenciales agentes con capacidad antiparasitaria. Revista de Biología Tropical, 68(4), 1241-1249.

Los compuestos naturales representan un papel importante en el descubrimiento de fármacos y el desarrollo de nuevas terapias medicinales, pues, se ha demostrado que las propiedades bioactivas de los productos de plantas medicinales se atribuyen principalmente a los compuestos fitoquímicos (Altemimi, Lakhssassi, Baharlouei, Watson, \& Lightfoot, 2017). Existen informes que indican efectos antiparasitarios de algunos de los compuestos 
bioactivos naturales tales como el alcaloide berberina (Ber), siendo uno de los metabolitos más estudiados, el cual posee actividad contra una variedad microorganismos, hongos, virus y parásitos (Gull, Anwar, Sultana, Alcayde, \& Nouman, 2015). El polifenol curcumina (Cur), actualmente, está recibiendo considerable atención por sus beneficios para la salud y la capacidad de prevenir las enfermedades de la civilización moderna, como los trastornos cardiovasculares o diabéticos antitumorales y además posee actividad antihelmíntica. La curcumina ha sido ampliamente estudiada ya que posee propiedades en contra de Plasmodium berghei y Schistosoma mansoni además de poseer actividad sobre Biomphalaria pfeifferi caracol y hospedador intermediario de $S$. mansoni (Spencer, Peña-Quintero, Canudas, Bujosa, \& Urdaneta, 2018). La quercetina (Qr) es uno de los bioflavonoides más abundante en la naturaleza, el cual es un excelente antioxidante y es conocida por sus efectos antiinflamatorios, antihipertensivos, vasodilatadores, antibacterianas, y principalmente antioxidante (Sharifi-Rad, Epifano, Fiorito, \& Álvarez-Suarez, 2020).

Las parasitosis son un problema que afecta gran cantidad de la población humana. Los antiparasitarios derivados de fuentes naturales, pueden ser una alternativa para el tratamiento de infecciones parasitarias. Existen distintos tipos de parásitos que afectan al humano, los cuales están vinculados estrechamente con la pobreza, aislamiento socio-geográfico y la falta de financiamiento para su atención. Entre las parasitosis más comunes se encuentra la amebiasis, la tricomoniasis y la estrongiloidiasis (Pozio, 2020).

Entamoeba histolytica es el causante principal de la amebiasis. Este parásito tiene la capacidad de penetrar en la mucosa intestinal para causar colitis amebiana y propagarse a través de la circulación portal a otros órganos, típicamente el hígado y el cerebro. La infección por este parásito es responsable de 50 millones de casos de amebiasis y 100000 muertes anualmente (Dingsdag \& Hunter, 2018). El metronidazol es el fármaco de elección para el tratamiento de la amebiasis, el cual tiene más de 25 años de uso y hoy en día se conoce que E. histolytica ya ha mostrado resistencia a este fármaco (Ghosh, Aycock, \& Schwebke, 2018). Trichomonas vaginalis causa la tricomoniasis la cual es la enfermedad de transmisión sexual no viral más común en el mundo. La tricomoniasis se ha asociado con secuelas graves, las más notables son en mujeres embarazadas, el tratamiento de elección se basa en el uso de metronidazol en dosis altas (2-4 g), durante 7 a 14 días (Lee \& Ryu, 2019). La estrongiloidiasis es una enfermedad causada por nemátodos del género Strongyloides presentes en áreas tropicales y subtropicales con clima adecuado para la supervivencia de las etapas larvarias de las especies de este género. Se estima que entre 30 y 100 millones de personas están infectadas en todo el mundo. La ivermectina (IV) es el fármaco de referencia contra la estrongiloidiasis, pero ya se han registrado casos de cepas resistentes (Andersen et al., 2019) and the Global Program to Eliminate LF delivers mass drug administration (MDA).

Conforme a los antecedentes mencionados, los objetivos del presente estudio fueron evaluar la actividad antiparasitaria in-vitro de berberina, curcumina y quercetina contra trofozoítos de E. histolytica, T. vaginalis y larvas de $S$. venezuelensis. Además, evaluar sus capacidades antioxidantes, hemolíticas y citoprotectoras.

\section{MATERIALES Y MÉTODOS}

Reactivos: 2,2'-Azobis(2-methylpropionamidine) dihydrochloride (AAPH), 2,2-diphenyl-1-picrylhydrazyl (DPPH), Berberina (Ber), Curcumina (Cur), Dimetilsulfóxido (DMSO), Ácido-etilendiaminotetraacético (EDTA), Ivermectina (IV), Metanol absoluto (MeOH), Metronidazol (Met), Quercetina (Qr) y Vitamina E (VitE) se adquirieron en Sigma-Aldrich ${ }^{\circledR}$ (Merck-Chemical ${ }^{\circledR}$, USA), el suero fetal bovino (SFB) se adquirió en Invitrogen, USA. Todos los reactivos fueron grado analítico.

Cepas: E. histolytica HM1-IMSS y $T$. vaginalis GT15 en medio PEHPS fueron 
proporcionada por el Centro de Investigación Biomédica del Noreste del IMSS. Las larvas filariformes (L3) de S. venezuelensis fueron proporcionadas por el Centro de Investigación de Enfermedades Tropicales de la Universidad de Salamanca, España, las cuales fueron obtenidas mediante el método de Baermann, de heces de ratas infectadas.

Ensayos de actividad biológica: En tubos de microcentrífuga (Eppendorf ${ }^{\circledR}$, Germany), se prepararon soluciones madre Ber, Cur y Qr $(1 \mathrm{mg} / \mathrm{ml})$ en DMSO el cual nunca fua mayor al $2 \% \mathrm{p} / \mathrm{v}$. Estas soluciones se esterilizaron con filtros de membrana de nylon de 0.22 $\boldsymbol{\mu} \mathrm{m}$ (Millipore-Merck ${ }^{\circledR}$, Germany), a partir de estas, se hicieron las soluciones de trabajo (0.5 a $200 \mu \mathrm{M}$ ) para determinar las $\mathrm{IC}_{50}$.

Para los ensayos con E. histolytica y $T$. vaginalis las soluciones se depositaron en viales de vidrio de $2 \mathrm{ml}$ estériles, los cuales contenían una suspensión de trofozoítos de $E$. histolytica $\left(2 \times 10^{4}\right.$ trofozoitos $\left./ \mathrm{ml}\right)$ o $T$. vaginalis $\left(1 \times 10^{5}\right.$ trofozoítos $\left./ \mathrm{ml}\right)$ en fase logarítmica, en medio PEHPS + SFB-10 \% y se incubaron a $37{ }^{\circ} \mathrm{C}$ durante $72 \mathrm{~h}$ y $24 \mathrm{~h}$ respectivamente. Después del tiempo de incubación los viales se enfriaron $\left(4{ }^{\circ} \mathrm{C} / 20 \mathrm{~min}\right)$ para que los trofozoítos se despegaran, se realizó una tinción con azul tripán al $10 \%$ y se realizó una dilución 1:10 con formalina para fijar, contar y medir la viabilidad de los trofozoítos, con un hemocitómetro. Como control positivo se utilizó metronidazol $(10 \mu \mathrm{M})$ y como control negativo solo medio de cultivo. En cuanto a los ensayos con L3 de $S$. venezuelensis, en una microplaca de 96 pozos se agregaron $100 \mu \mathrm{l}$ con 150 larvas por pozo las cuales se preincubaron $30 \mathrm{~min}$ ( 28 ${ }^{\circ} \mathrm{C}$ ) y finalmente se agregaron $100 \mu \mathrm{l}$ de los tratamientos previamente descritos. Como control positivo se usó IV $(10 \mu \mathrm{M})$ y como control negativo agua destilada (Legarda-Ceballos et al., 2016), el ensayo se llevó a cabo durante 72 $\mathrm{h}$ a $28{ }^{\circ} \mathrm{C}$ y la viabilidad fue evaluada directamente mediante el registro del movimiento larval después de la estimulación con luz natural directa durante dos minutos utilizando un microscopio invertido (CK2, Olympus, Japón), las larvas se consideraron muertas cuando no se detectó movimiento durante al menos dos minutos de examen detallado.

Actividad antioxidante: Se usó el método de reducción del radical DPPH. Los tratamientos $(\mathrm{Tr})$ fueron evaluados a concentraciones de 20-2 $500 \mu \mathrm{g} / \mathrm{ml}$. El DPPH fue preparado a $125 \mu \mathrm{M}$ en $\mathrm{MeOH}$ absoluto, se tomaron $100 \mu \mathrm{l}$ de cada concentración respectiva y se agregaron $100 \mu \mathrm{l}$ del DPPH. Las muestras se dejaron reposar por $30 \mathrm{~min}$ en ausencia de luz (Rodríguez-Magaña et al., 2019). La absorbancia (Abs) fue medida a $517 \mathrm{~nm}$ con un espectrofotómetro Genesys (Thermo-Scientific ${ }^{\mathrm{TM}}$, USA). Como control positivo $(\mathrm{C}+)$, se utilizó una solución de VitE y como blanco (Bco) $\mathrm{MeOH}$; el porcentaje de reducción se calculó utilizando la fórmula:

$$
\% \text { Reducción }=[(\mathrm{AbsBco}-\mathrm{AbsTr}) /(\mathrm{AbsBco})] \times 100
$$

Ensayo de hemólisis y citoprotección en eritrocitos humanos: Se recolecto sangre de donadores sanos y el suero fue separado. Los eritrocitos, se lavaron con EDTA $(1.5 \mathrm{mg} / \mathrm{ml}$ de sangre), se mezclaron y centrifugaron (1 000 rpm por $4 \mathrm{~min}$ ) a temperatura ambiente. Después, la suspensión celular es lavada y centrifugada cuatro veces en solución amortiguadora de fosfatos en proporción 1:1 (PBS $10 \mathrm{mM}$ a $\mathrm{pH}$ 7.4) eliminando los sobrenadantes y se prepara una suspensión al $5 \%$ v/v en PBS. Para la evaluación de la hemólisis, la suspensión se incubó con diferentes concentraciones de Ber, Cur o Qr, y de soluciones testigo previamente preparadas en $\mathrm{MeOH}$ absoluto $(50$ a $1000 \mu \mathrm{g} /$ $\mathrm{ml}$ ) en viales de $2 \mathrm{ml}$ durante $30 \mathrm{~min}$ a $37{ }^{\circ} \mathrm{C}$ y en ausencia de luz, estos fueron catalogados como tratamientos ( $\mathrm{Tr}$ ). Como control negativo (C-) se utilizó eritrocitos sin tratamiento y como control positivo $(\mathrm{C}+)$ eritrocitos con agua destilada estéril para producir la hemólisis osmótica en los eritrocitos (Elizondo-Luevano et al., 2020). Para la evaluación del efecto citoprotector, a los tratamientos se les añadió AAPH (150 $\mathrm{mM}$ en PBS) más la suspensión de eritrocitos y se incubaron a $37^{\circ} \mathrm{C}$ ( $5 \mathrm{~h}$ a $\left.200 \mathrm{rpm}\right)$ utilizando 
una incubadora de rotación MaxQ $\mathrm{Q}^{\mathrm{TM}}$ (ThermoScientific $^{\mathrm{TM}}$, USA), en ausencia de luz. El control negativo (C-), consistió en PBS con la suspensión de eritrocitos sin incluir AAPH y como control positivo $(\mathrm{C}+)$, la suspensión de eritrocitos con AAPH. Pasado el tiempo de incubación, los tratamientos se centrifugaron a $13000 \mathrm{rpm}$ por $4 \mathrm{~min}$ a $4{ }^{\circ} \mathrm{C}$, se tomaron $200 \mu \mathrm{l}$ del sobrenadante y se pusieron en una microplaca transparente de 96 pozos con fondo plano. La hemólisis se determinó mediante la lectura a $540 \mathrm{~nm}$, con un lector de microplacas $\mathrm{EPOCH}^{\mathrm{TM}}$ (BioTek ${ }^{\mathrm{TM}}$, USA), para registrar la absorbancia de cada tratamiento menos la absorbancia presentada por el vehículo (AbsTr) y finalmente, se calculó el porcentaje de hemólisis $=([(\mathrm{AbsTr}-\mathrm{AbsC}-) /(\mathrm{AbsC}+-\mathrm{AbsC}-)] \times$ 100) y citoprotección $=([1-(\mathrm{AbsTr}-\mathrm{AbsC}-) /$ $(\mathrm{AbsC}+-\mathrm{AbsC}-)] \times 100)$.

Análisis estadístico: La prueba de análisis de varianza (Anova) de una vía se usó para determinar si existía diferencia significativa entre las concentraciones evaluadas y la prueba honesta de significancia Tukey para determinar la diferencia entre las medias en los tratamientos evaluados. La concentración inhibitoria media $\left(\mathrm{IC}_{50}\right)$ y la concentración efectiva media
$\left(\mathrm{CE}_{50}\right)$ se determinó por la prueba de Probit, con un intervalo de confianza del $95 \%$. Los análisis se realizaron con el software SPSS, versión 23.0 (SPSS, Inc. USA). Las diferencias se consideraron significativas a $\mathrm{P}<0.05$. Todos los ensayos se realizaron por triplicado.

\section{RESULTADOS}

Actividad biológica: Berberina, curcumina y quercetina in-vitro mostraron actividades significativas $(\mathrm{P}<0.05)$ contra E. histolytica, $T$. vaginalis y $S$. venezuelensis (Tabla 1). Mediante la prueba de Tukey, se puede observar que no existe diferencia significativa $(\mathrm{P}>0.05)$ entre Ber y los fármacos control contra $E$. histolytica y $S$. venezuelensis; sin embargo, en contra de T. vaginalis si hubo diferencia significativa. También se observa que Cur y Qr, no fueron tan efectivos, en comparación con los fármacos control para los tres parásitos ensayados, ya que mostraron alta diferencia significativa $(\mathrm{P}<0.001)$.

Capacidad antioxidante: Se observó un diferente desempeño en la capacidad antioxidante de cada uno de los metabolitos (Qr $>$ VitE $>$ Cur $>$ Ber), siendo Qr el más efectivo, incluso

TABLA 1

Actividad in-vitro de berberina, curcumina y quercetina sobre Entamoeba histolytica, Trichomona vaginalis y Strongyloides venezuelensis

TABLE 1

In-vitro activity of berberine, curcumin and quercetin on Entamoeba histolytica, Trichomona vaginalis and Strongyloides venezuelensis

\begin{tabular}{|c|c|c|c|}
\hline \multirow{2}{*}{ Tratamientos } & \multicolumn{3}{|c|}{$\mathrm{IC}_{50}(\mu \mathrm{M})$} \\
\hline & E. histolytica & T. vaginalis & S. venezuelensis \\
\hline Control (Met) & $1.0 \pm 0.2^{\mathrm{a}}$ & $0.05 \pm 0.0^{\mathrm{a}}$ & - \\
\hline Control (IV) & - & - & $1.3 \pm 0.2^{\mathrm{a}}$ \\
\hline Ber & $1.7 \pm 0.5^{\mathrm{a}}$ & $1.2 \pm 0.1^{b}$ & $1.9 \pm 0.3^{\mathrm{a}}$ \\
\hline Cur & $55.3 \pm 1.1^{\mathrm{b}}$ & $40.6 \pm 3.2^{\mathrm{c}}$ & $13.7 \pm 2.1^{\mathrm{b}}$ \\
\hline Qr & $147.2 \pm 3.9^{\mathrm{c}}$ & $93.2 \pm 2.6^{\mathrm{d}}$ & $110.9 \pm 4.4^{\mathrm{c}}$ \\
\hline P Anova & $<0.001$ & $<0.001$ & $<0.001$ \\
\hline F Anova & 5.20 & 16.3 & 17.4 \\
\hline
\end{tabular}

Los valores se muestran como la Media $\pm \mathrm{DE}(\mathrm{P}<0.05)$. Letras diferentes dentro de la misma columna son significativamente diferentes, analizados vía la prueba de Tukey.

Values are shown as mean $\pm \mathrm{SD}(\mathrm{P}<0.05)$. Different letters within the same column are significantly different analyzed via the Tukey test. 
en comparación con el control positivo VitE (P $<0.001)$. La prueba de Tukey arrojó que existe diferencia significativa entre los tratamientos, excepto entre Cur y VitE ( $\mathrm{P}>0.05)$, los cuales tuvieron capacidad antioxidante similar, pues estos no presentaron diferencia entre sus medias (Tabla 2).

TABLA 2

Capacidad de captación del radical DPPH

TABLE 2

DPPH radical scavenging capacity

\begin{tabular}{cc} 
Tratamientos & $\mathrm{EC}_{50}(\mu \mathrm{g} / \mathrm{ml})$ \\
\hline VitE & $16.9 \pm 1.4^{\mathrm{b}}$ \\
Ber & $42.0 \pm 6.5^{\mathrm{c}}$ \\
Cur & $20.1 \pm 0.8^{\mathrm{b}}$ \\
Qr & $1.1 \pm 0.1^{\mathrm{a}}$ \\
\hline
\end{tabular}

Los valores se muestran como la Media \pm DE $(\mathrm{P}<$ 0.05). Letras diferentes dentro de la misma columna son significativamente diferentes, analizados vía la prueba de Tukey.

Values are shown as mean $\pm \mathrm{SD}(\mathrm{P}<0.05)$. Different letters within the same column are significantly different analyzed via the Tukey test.
Actividad hemolítica y citoprotectora: La actividad hemolítica de Qr in-vitro mostró que este metabolito no tiene capacidad lítica significativa de los hematíes $(\mathrm{P}=0.264)$, en comparación con el control no tratado, el cual no presentó hemólisis. Para Ber y Cur se presentaron altas diferencia significativa $(\mathrm{P}$ $<0.001$ ) en comparación con dicho control (Tabla 3). Además se pudo observar que con Ber, que la hemolisis va en aumento conforme aumenta la concentración. Para Cur y Qr no se presentaron altas toxicidades, siendo las hemólisis del 1.9 y $0.7 \%$ respectivamente, las exhibidas la máxima concentración evaluada y sin toxicidad significativa a la concentración más baja. La actividad citoprotectora in-vitro de Ber, Cur y Qr, presentaron diferencia significativa $(\mathrm{P}<0.05)$, en comparación con el control sin protección de los eritrocitos. Se puedo observar que Ber no fue tan eficiente como Cur y Qr, ya que estos metabolitos a partir de 50 $\mu \mathrm{g} / \mathrm{ml}$, mostraron citoprotección del 71 y 89 $\%$ respectivamente, en comparación al control, mientras que Ber presentó $2.2 \%$.

TABLA 3

Evaluación de la hemólisis y citoprotección de berberina, curcumina y quercetina

TABLE 3

Evaluation of the hemolysis and cytoprotection of berberine, curcumin and quercetin

\begin{tabular}{ccccccc}
$\mu \mathrm{c} / \mathrm{ml}$ & \multicolumn{3}{c}{ Hemólisis } & \multicolumn{3}{c}{ Citoprotección } \\
& Ber & Cur & Qr & Ber & Cur & Qr \\
Control & $0.0 \pm 0.0^{\mathrm{a}}$ & $0.0 \pm 0.0^{\mathrm{a}}$ & $0.0 \pm 0.0^{\mathrm{a}}$ & $0.0 \pm 0.0^{\mathrm{e}}$ & $0.0 \pm 0.0^{\mathrm{e}}$ & $0.0 \pm 0.0^{\mathrm{d}}$ \\
50 & $1.0 \pm 0.1^{\mathrm{b}}$ & $0.5 \pm 0.1^{\mathrm{a}}$ & $0.0 \pm 0.0^{\mathrm{a}}$ & $2.2 \pm 0.0^{\mathrm{d}}$ & $71.0 \pm 2.1^{\mathrm{d}}$ & $89.2 \pm 2.0^{\mathrm{c}}$ \\
100 & $2.4 \pm 0.4^{\mathrm{b}}$ & $0.9 \pm 0.2^{\mathrm{b}}$ & $0.0 \pm 0.0^{\mathrm{a}}$ & $3.7 \pm 0.0^{\mathrm{c}}$ & $79.4^{\mathrm{a}} \pm 3.4^{\mathrm{c}}$ & $90.5 \pm 1.8^{\mathrm{c}}$ \\
200 & $8.8 \pm 1.6^{\mathrm{c}}$ & $1.1 \pm 0.2^{\mathrm{b}}$ & $0.1 \pm 0.0^{\mathrm{b}}$ & $7.4 \pm 0.1^{\mathrm{a}}$ & $81.1 \pm 2.0^{\mathrm{c}}$ & $94.3 \pm 3.1^{\mathrm{b}}$ \\
400 & $19.8 \pm 1.2^{\mathrm{d}}$ & $1.2 \pm 0.1^{\mathrm{b}}$ & $0.1 \pm 0.0^{\mathrm{b}}$ & $5.8 \pm 0.0^{\mathrm{b}}$ & $93.7 \pm 4.7^{\mathrm{b}}$ & $96.8 \pm 3.6^{\mathrm{b}}$ \\
600 & $32.9 \pm 2.1^{\mathrm{e}}$ & $1.3 \pm 0.1^{\mathrm{b}}$ & $0.2 \pm 0.0^{\mathrm{c}}$ & $2.5 \pm 0.1^{\mathrm{d}}$ & $98.3 \pm 2.3^{\mathrm{a}}$ & $100 \pm 0.0^{\mathrm{a}}$ \\
800 & $67.9 \pm 2.7^{\mathrm{f}}$ & $1.5 \pm 0.2^{\mathrm{b}}$ & $0.4 \pm 0.1^{\mathrm{d}}$ & $2.1 \pm 0.1^{\mathrm{d}}$ & $100 \pm 0.0^{\mathrm{a}}$ & $100 \pm 0.0^{\mathrm{a}}$ \\
1000 & $84.7 \pm 6.0^{\mathrm{g}}$ & $1.9 \pm 0.3^{\mathrm{c}}$ & $0.7 \pm 0.1^{\mathrm{e}}$ & $0.7 \pm 0.1^{\mathrm{e}}$ & $100 \pm 0.0^{\mathrm{a}}$ & $100 \pm 0.0^{\mathrm{a}}$ \\
SE & 11.5 & 0.2 & 0.1 & 0.9 & 4.4 & $<.7$ \\
P Anova & $<0.001$ & $<0.001$ & 0.264 & $<0.05$ & $<0.001$ & $<0.001$ \\
F Anova & 3.866 & 17.177 & 1.646 & 61.461 & 35.899 & 3.865 \\
\hline
\end{tabular}

Valores mostrados como la media $\pm \mathrm{DE}(\mathrm{P}<0.05)$ del porcentaje de hemólisis o citoprotección, más el error estándar $(\mathrm{SE})$. Letras diferentes dentro de la misma columna son significativamente diferentes, analizados vía la prueba de Tukey.

Values shown as the mean $\pm \mathrm{SD}(\mathrm{P}<0.05)$ of the percentage of hemolysis or cytoprotection, plus the standard error (SE). Different letters within the same column are significantly different, analyzed via the Tukey test. 


\section{DISCUSIÓN}

Los resultados obtenidos en este estudio indican que berberina, curcumina y quercetina poseen actividad en contra de la viabilidad de Entamoeba histolytica, Tricomonas vaginalis y Strongyloides venezuelensis in-vitro (Tabla 1). En cuanto a la actividad biológica destaca Ber siendo el más efectivo, arrojando $\mathrm{IC}_{50}$ de 1.7, 1.2 y $1.9 \mu \mathrm{M}$ en contra de E. histolytica, $T$. vaginalis y $S$. venezuelensis respectivamente. Lo que indica que posiblemente la estructura química alcaloide favorece la actividad biológica. Existen estudios en productos naturales con extractos ricos en este metabolito que muestran este comportamiento (Elizondo-Luévano et al., 2018). Se ha demostrado que Ber ejerce efectos inhibidores contra G. lamblia, T. vaginalis y $E$. histolytica, en los que se observó que los trofozoítos se hincharon con presencia de depósitos de glucógeno y con la aparición de vacuolas de forma irregular en el citoplasma con cambios morfológicos en los parásitos, incluyendo agregación de cromatina en el núcleo y agregaciones de pequeñas vacuolas en el citoplasma (Shen, Jiang, Yang, Wang, \& Zhu, 2017). Al momento se tienen estudios de la berberina en contra de $S$. mansoni, Echinococcus multilocularis y E. granulosus pero no sobre S. venezuelensis. En dicho estudio Ber mostró ser significativamente igual de eficaz que el fármaco control (Siles-Lucas, Casulli, Cirilli, \& Carmena, 2018), muy similar a lo encontrado en nuestro estudio donde se comporta significativamente igual que los fármacos control (metronidazol e ivermectina).

En este contexto, este estudio mostró que moléculas que no pertenecen a la familia de los alcaloides presentan buena actividad biológica en contra de $E$. histolytica, $T$. vaginalis y $S$. venezuelensis a dosis relativamente bajas $\left(\mathrm{IC}_{50}\right.$ con $\mathrm{IC}_{50} 55.3,40.6$ y $13.7 \mu \mathrm{M}$ respectivamente) como es el caso de curcumina un conocido antioxidante. A diferencia de Ber con esta molécula no existen muchos estudios de su actividad contra parásitos, se conocen más algunos efectos sobre ciertos microorganismos y hongos (Aquino et al., 2016; da Silva et al., 2020).
Existen reportes que indican que en concentraciones de hasta $50 \mu \mathrm{M}$ atenúan la virulencia de E. histolytica sin afectar el crecimiento de trofozoítos ni desencadenar daño hepático, además disminuye la expresión de genes asociados con su virulencia (lectina gal/galnac, ehcp1 y ehcp5) lo cual fue correlacionado con una invasión amebiana significativamente menor (Rangel-Castañeda et al., 2019). Existen otros estudios con Cur en contra de $S$. mansoni en fase adulta y se reporta un $100 \%$ de letalidad a $50 \mu \mathrm{M}$, donde se observó reducción de la oviposición, separación inducida de machos y hembras y reducción de la actividad motora (Ndjonka, Rapado, Silber, Liebau, \& Wrenger, 2013).

Por otro lado, las afecciones por parásitos están relacionadas con el estrés oxidativo, por ejemplo de forma crítica, en la etiopatología del absceso hepático amebiano. Por tal motivo, este estudio también se evaluó la capacidad antioxidante y los resultados proporcionaron una clara evidencia que los agentes antiparasitarios pueden además ser antioxidantes, pero que no necesariamente amabas actividades se encuentran relacionadas. Así, en esta investigación se compararon las actividades antioxidantes de los metabolitos estudiados contra VitE, control en este tipo de ensayos, debido a su alta actividad antioxidante. Se observó que el mejor antiparasitario, Ber, fue el compuesto menos efectivo como antioxidante $\left(\mathrm{EC}_{50} 42\right.$ $\mu \mathrm{g} / \mathrm{ml})$, por otro lado, Qr resultó ser altamente antioxidante $\left(\mathrm{EC}_{50} 1.1 \mu \mathrm{g} / \mathrm{ml}\right)$, incluso presentó actividad superior a la VitE $\left(\mathrm{EC}_{50} 16.9 \mu \mathrm{g} / \mathrm{ml}\right)$, sin embargo, este compuesto prácticamente no presentó actividad contra los parásitos. Cabe mencionar, que esta falta de actividad no puede adjudicarse a la incapacidad de penetración ya que ha sido reportado que Qr posee actividad intracelular como antioxidante debido a sus efectos sobre el glutatión (GSH), la actividad enzimática, las vías de transducción de señales y las especies reactivas de oxígeno (ROS) causadas por factores ambientales y toxicológicos, además de que mantiene el equilibrio oxidativo en la célula (Xu, Hu, Wang, \& Cui, 2019). 
Los ensayos de toxicidad demostraron que la hemólisis causada por Ber fue directamente proporcional a la concentración. Se destaca que a concentraciones menores a 2 $\mu \mathrm{M}$, esta molécula es efectiva en contra de los parásitos evaluados, y está muy por debajo de la concentración hemolítica en concordancia con otros estudios En cuanto a Cur y Qr, estos no presentaron diferencia significativa entre ellos $(\mathrm{P}>0.05)$, resultando en una hemolisis menor al $2 \%$ inclusive en la concentración más alta evaluada, por consiguiente, podrían ser clasificados como no tóxicos, ya que la posible hemólisis presentada es relacionada al estrés mecánico al momento de la agitación durante el periodo de incubación (Pozzo et al., 2020). Estos resultados podrían sugerir que las actividades antiparasitaria y de toxicidad celular se relacionan y que presumiblemente el efecto antioxidante está más relacionado con la inocuidad. Además la literatura menciona que compuestos alcaloides como Ber pueden ser tóxicos en altas concentraciones y que incluso puede llegar a intercalarse en el ADN. En contraste, compuestos con grupos fenólicos como Cur y Qr, poseen una alta actividad citoprotectora (Pongkittiphan, Chavasiri, \& Supabphol, 2015). En este estudio dicha actividad se evaluó mediante la descomposición térmica del AAPH, ya que es un modelo efectivo para estudiar la inhibición de radicales libres por antioxidantes. Esta prueba es ampliamente utilizada para la evaluación de la disrupción de las membranas celulares inducida por radicales libres de oxígeno (Pozzo et al., 2020). Los resultaron mostraron que efectivamente aquellos compuestos que se habían destacado como antioxidantes, es decir Qr y Cur, brindaron una mejor protección $(\mathrm{P}<0.001)$ en comparación con Ber, ya que éste no alcanzó más de 3.5 $\%$ de protección. Diferentes investigaciones, mencionan que la resistencia de los eritrocitos al estrés oxidativo es debida a la presencia de polifenoles o flavonoides, ya que obstaculizan la difusión de los radicales libres, disminuyendo en gran manera las reacciones de oxidación (Caddeo et al., 2019). Finalmente, los resultados en cuales Cur y Qr no provocaron hemólisis significativa pero presentaron protección sobre la membrana eritrocitaria, aunados a aquellos que demuestran que Ber no presentó protección sobre los eritrocitos, indican que posiblemente la acción biológica de Ber no es ejercida a nivel de membrana, sino dentro de la célula (Hatia et al., 2014).

Existe un renovado interés en los productos naturales como punto de partida para el descubrimiento de medicamentos para combatir las parasitosis, ya que son escasos los recursos para tratarlas (Yones et al., 2016). Por lo cual es necesario desarrollar nuevos medicamentos contra estas enfermedades que afectan al hombre mundialmente. El uso de productos de origen naturales para el tratamiento de las parasitosis va en aumento, esto debido a que el control y tratamiento, está basado en la aplicación de los mismos medicamentos desde hace varias décadas, es por tal motivo el aumento en el número de investigaciones para el uso de diversas sustancias de origen natural, ya sea vegetal o animal (Hayat, Azam, \& Shin, 2016). En la presente investigación se demostró que Ber, Cur y Qr, prometedoras en cuanto a la inhibición de la viabilidad de E. histolytica, $T$. vaginalis y $S$. venezuelensis además poseen propiedades antioxidante y citoprotectora. El mecanismo de la actividad de Ber, Cur y Qr, aún no están elucidados, se requieren experimentos en distintas etapas de los parásitos, además de los mecanismos de acción en esas etapas y experimentos en modelos in-vivo, para dilucidar los mecanismos de acción. Así mismo, estos estudios podrán llegar a validar el uso de extractos vegetales donde posiblemente las acciones combinadas resulten en beneficios terapéuticos que deberían explorase.

Declaración de ética: Los autores declaran estar de acuerdo con esta publicación y se han hecho aportes que justifican su autoría. No hay conflicto de interés y se han cumplido los procedimientos éticos pertinentes. El financiamiento viene detallado en la sección de agradecimientos. Los procedimientos empleados cumplen las normas de la Unión Europea y Mexicanas (No.reg.PAE/SA/001). 


\section{AGRADECIMIENTOS}

Al Consejo Nacional de Ciencia y Tecnología (CONACYT) por el apoyo brindado (Proyecto CB176853) a J.H. Elizondo-Luevano (No.reg.418935) y a O.A. Pérez-Narváez (No. reg.745689).

\section{RESUMEN}

Introducción: El uso indiscriminado de agentes antiparasitarios ha resultado en el establecimiento de resistencia a ellos. Por lo cual es necesario el desarrollo de nuevas alternativas de tratamiento. Los productos naturales poseen diversas cualidades como posibles coadyuvantes en terapias contra distintos agentes etiológicos, entre los que destaca sus efectos antiparasitarios. Objetivo: Evaluar la actividad antiparasitaria, antioxidante, citotóxica y citoprotectora de Berberina (Ber), Curcumina (Cur) y Quercetina (Qr). Metodología: Se prepararon soluciones de Ber, Cur y Qr grado analítico y se realizaron alícuotas a diferentes concentraciones para su evaluación en contra de: Entamoeba histolytica, Trichomonas vaginalis y Strongyloides venezuelensis, para ello, se determinó la concentración inhibitoria media $\left(\mathrm{IC}_{50}\right)$, además se determinó la capacidad antioxidante $\left(\mathrm{CE}_{50}\right)$ mediante la prueba de DPPH, ambos por la prueba de Probit. Mediante la técnica de hemólisis se determinó la actividad citotóxica y citoprotectora, se aplicó Anova y la prueba de Tukey para determinar la diferencia de las medias en los tratamientos evaluados. Resultados: Ber, Cur y Qr, presentaron actividad en contra de E. histolytica, T. vaginalis y $S$. venezuelensis in-vitro. Ber presentó $\mathrm{IC}_{50}$ de $1.7,1.2$ y 1.9 $\mu \mathrm{M}$ respectivamente siendo más efectivo en comparación de Cur con $\mathrm{IC}_{50}$ de 55.3, 40.6 y $13.7 \mu \mathrm{M}$ o Qr con $\mathrm{IC}_{50}$ de 147.2, 93.2 y $110.9 \mu \mathrm{M}$, sin embargo, la mejor actividad antioxidante $\left(\mathrm{EC}_{50}=1.1 \mu \mathrm{g} / \mathrm{ml}\right)$, citoprotectora y menos hemolítica, fue presentada por $\mathrm{Qr}(\mathrm{P}<0.001)$ en comparación con el control evaluado. Conclusiones: Los metabolitos de origen natural berberina, curcumina y quercetina, poseen actividad en contra de trofozoítos de E. histolytica, $T$. vaginalis y larvas de $S$. venezuelensis en dosis bajas comparables con los fármacos de referencia para el caso de Ber. Además, estos productos de origen natural, no sintético podrían ser objeto de futuras investigaciones para coadyuvar al tratamiento de parasitosis, ya que, en dosis bajas, mostraron actividad antioxidante sin mostrar hemólisis considerable en eritrocitos humanos.

Palabras clave: actividad antiparasitaria; antiparasitarios; berberina; curcumina; quercetina.

\section{REFERENCIAS}

Altemimi, A., Lakhssassi, N., Baharlouei, A., Watson, D.G., \& Lightfoot, D.A. (2017). Phytochemicals: Extraction, Isolation, and Identification of Bioactive Compounds from Plant Extracts. Plants, 6(4), 42.

Andersen, B.J., Rosa, B.A., Kupritz, J., Meite, A., Serge, T., Hertz, M.I., ...Weil, G.J. (2019). Systems analysisbased assessment of post-treatment adverse events in lymphatic filariasis. PLoS Neglected Tropical Diseases, 13(9), 1-27.

Aquino, P., Gomes-Figueredo, F., Pereira, N., Nascimento, E., Martin, A., Veras, H., ... Menezes, I. (2016). Avaliação da atividade anti-inflamatória tópica e antibacteriana do extrato metanólico das folhas de Sideroxylon obtusifolium. Acta Biológica Colombiana, 21(1), 131-140.

Caddeo, C., Gabriele, M., Fernández-Busquets, X., Valenti, D., Fadda, A.M., Pucci, L., \& Manconi, M. (2019). Antioxidant activity of quercetin in Eudragit-coated liposomes for intestinal delivery. International Journal of Pharmaceutics, 565, 64-69.

da Silva, A.F., da Rocha, C.Q., da Silva, L., Carvalho Júnior, A.R., Mendes, I., de Araruna, A.B., ... Monteiro, C.A. (2020). Antifungal and Antivirulence Activities of Hydroalcoholic Extract and Fractions of Platonia insignis Leaves against Vaginal Isolates of Candida Species. Pathogens, 9(2), 84.

Dingsdag, S.A., \& Hunter, N. (2018). Metronidazole: an update on metabolism, structure-cytotoxicity and resistance mechanisms. The Journal of Antimicrobial Chemotherapy, 73(2), 265-279.

Elizondo-Luévano, J.H., Castro-Ríos, R., Sánchez-García, E., Hernández-García, M.E., Vargas-Villarreal, J., Rodríguez-Luis, O.E., \& Chávez-Montes, A. (2018). In Vitro Study of Antiamoebic Activity of Methanol Extracts of Argemone mexicana on Trophozoites of Entamoeba histolytica HM1-IMSS. The Canadian Journal of Infectious Diseases \& Medical Microbiology = Journal Canadien Des Maladies Infectieuses et de La Microbiologie Medicale, 2018, 7453787.

Elizondo-Luevano, J.H., Verde-Star, J., González-Horta, A., Castro-Ríos, R., Hernández-García, M.E., \& Chávez-Montes, A. (2020). In Vitro Effect of Methanolic Extract of Argemone mexicana against Trichomonas vaginalis. The Korean Journal of Parasitology, 58(2), 135-145.

Ghosh, A.P., Aycock, C., \& Schwebke, J.R. (2018). In Vitro Study of the Susceptibility of Clinical Isolates of Trichomonas vaginalis to Metronidazole and Secnidazole. Antimicrobial Agents and Chemotherapy, 62(4), e02329-17. 
Gull, T., Anwar, F., Sultana, B., Alcayde, M.A.C., \& Nouman, W. (2015). Capparis species: A potential source of bioactives and high-value components: A review. Industrial Crops and Products, 67, 81-96.

Hatia, S., Septembre-Malaterre, A., Le Sage, F., BadiouBénéteau, A., Baret, P., Payet, B., ... Gonthier, M.P. (2014). Evaluation of antioxidant properties of major dietary polyphenols and their protective effect on 3T3-L1 preadipocytes and red blood cells exposed to oxidative stress. Free Radical Research, 48(4), 387-401.

Hayat, F., Azam, A., \& Shin, D. (2016). Recent progress on the discovery of antiamoebic agents. Bioorganic and Medicinal Chemistry Letters, 26(21), 5149-5159.

Lee, J., \& Ryu, J.S. (2019). Current Status of Parasite Infections in Indonesia: A Literature Review. The Korean Journal of Parasitology, 57(4), 329-339.

Legarda-Ceballos, A.L., Rojas-Caraballo, J., López-Abán, J., Ruano, A.L., Yepes, E., Gajate, C., Mollinedo, F., \& Muro, A. (2016). The alkylphospholipid edelfosine shows activity against Strongyloides venezuelensis and induces apoptosis-like cell death. Acta Tropica, $162,180-187$.

Ndjonka, D., Rapado, L.N., Silber, A.M., Liebau, E., \& Wrenger, C. (2013). Natural products as a source for treating neglected parasitic diseases. International Journal of Molecular Sciences, 14(2), 3395-3439.

Pongkittiphan, V., Chavasiri, W., \& Supabphol, R. (2015). Antioxidant effect of berberine and its phenolic derivatives against human fibrosarcoma cells. Asian Pacific Journal of Cancer Prevention, 16(13), 5371-5376.

Pozio, E. (2020). How globalization and climate change could affect foodborne parasites. Experimental Parasitology, 208, 107807.

Pozzo, L., Russo, R., Frassinetti, S., Vizzarri, F., Árvay, J., Vornoli, A., ... Longo, V. (2020). Wild Italian Prunus spinosa L. Fruit Exerts In Vitro Antimicrobial Activity and Protects Against In Vitro and In Vivo Oxidative Stress. Foods, 9(1), 5.

Sharifi-Rad, M., Epifano, F., Fiorito, S., \& Álvarez-Suarez, J.M. (2020). Phytochemical analysis and biological investigation of Nepeta juncea Benth. different extracts. Plants, 9(5), 646.

Rangel-Castañeda, I.A., Carranza-Rosales, P., GuzmánDelgado, N.E., Hernández-Hernández, J.M., González-Pozos, S., Pérez-Rangel, A., \& Castillo-Romero, A. (2019). Curcumin attenuates the pathogenicity of Entamoeba histolytica by regulating the expression of virulence factors in an Ex-Vivo model Infection. Pathogens, 8(3), 127.

Rodríguez-Magaña, M.P., Cordero-Pérez, P., Rivas-Morales, C., Oranday-Cárdenas, M.A., Moreno-Peña, D.P., García-Hernández, D.G., \& Leos-Rivas, C. (2019). Hypoglycemic Activity of Tilia americana, Borago officinalis, Chenopodium nuttalliae, and Piper sanctum on Wistar Rats. Journal of Diabetes Research, 2019, 7836820.

Shen, C.Y., Jiang, J.G., Yang, L., Wang, D.W., \& Zhu, W. (2017). Anti-ageing active ingredients from herbs and nutraceuticals used in traditional Chinese medicine: pharmacological mechanisms and implications for drug discovery. British Journal of Pharmacology, 174(11), 1395-1425.

Siles-Lucas, M., Casulli, A., Cirilli, R., \& Carmena, D. (2018). Progress in the pharmacological treatment of human cystic and alveolar echinococcosis: Compounds and therapeutic targets. PLoS Neglected Tropical Diseases, 12(4), e0006422.

Spencer, L.M., Peña-Quintero, A., Canudas, N., Bujosa, I., \& Urdaneta, N. (2018). Antimalarial effect of two photo-excitable compounds in a murine model with Plasmodium berghei (Haemosporida: Plasmodiidae). Revista de Biología Tropical, 66(2), 880-891.

Xu, D., Hu, M.J., Wang, Y.Q., \& Cui, Y.L. (2019). Antioxidant activities of quercetin and its complexes for medicinal application. Molecules, 24(6), 1123.

Yones, D.A., Badary, D.M., Sayed, H.M., Bayoumi, S.A., Khalifa, A.A., \& El-Moghazy, A.M. (2016). Comparative Evaluation of Anthelmintic Activity of Edible and Ornamental Pomegranate Ethanolic Extracts against Schistosoma mansoni. BioMed Research International, 2016, 2872708. 